\title{
Positions and sizes of X-ray solar flare sources
}

\author{
E. P. Kontar and N. L. S. Jeffrey
}

\author{
Department of Physics and Astronomy, University of Glasgow, G12 8QQ, UK \\ e-mail: eduard@astro.gla.ac.uk
}

Received 14 January 2010 / Accepted 8 March 2010

\begin{abstract}
Aims. The positions and source sizes of X-ray sources taking into account Compton backscattering (albedo) are investigated. Methods. Using a Monte Carlo simulation of X-ray photon transport including photo-electric absorption and Compton scattering, we calculate the apparent source sizes and positions of X-ray sources at the solar disk for various source sizes, spectral indices and directivities of the primary source.

Results. We show that the albedo effect can alter the true source positions and substantially increase the measured source sizes. The source positions are shifted by up to $\sim 0.5^{\prime \prime}$ radially towards the disk centre and 5 arcsec source sizes can be two times larger even for an isotropic source (minimum albedo effect) at $1 \mathrm{Mm}$ above the photosphere. The X-ray sources therefore should have minimum observed sizes, and thus their FWHM source size (2.35 times second-moment) will be as large as 7" in the 20-50 keV range for a disk-centered point source at a height of $1 \mathrm{Mm}\left(\sim 1.4^{\prime \prime}\right)$ above the photosphere. The source size and position change is greater for flatter primary X-ray spectra, a stronger downward anisotropy, for sources closer to the solar disk centre, and between the energies of 30 and $50 \mathrm{keV}$.

Conclusions. Albedo should be taken into account when X-ray footpoint positions, footpoint motions or source sizes from e.g. RHESSI or Yohkoh data are interpreted, and we suggest that footpoint sources should be larger in X-rays than in either optical or EUV ranges.
\end{abstract}

Key words. Sun: flares - Sun: activity - Sun: X-rays, gamma rays - Sun: chromosphere

\section{Introduction}

Hard X-ray (HXR) emission produced via collisional bremsstrahlung from solar flares by non-thermal electrons is the primary diagnostic tool in the study of electron acceleration and transport. The spectral and spatial distributions of HXR sources can help us with vital clues to improve our current understanding of the underlying physics involved in energetic electron acceleration and transport. While recent (Hard X-ray Telescope (HXT) on Yohkoh, Kosugi et al. 1991), and modern (Reuven Ramaty High Energy Solar Spectroscopic Imager, Lin et al. 2002), solar HXR telescopes have achieved superb X-ray image resolutions, indirect imaging using either pairs of occultation grids on Yohkoh or rotating modulating collimators on RHESSI have (i) limited dynamic range and (ii) often provided inadequate spatial resolution of lengths exceeding the size of e.g. EUV footpoints or the vertical extent of the chromosphere. Thus RHESSI image resolution for the majority of solar flares is limited to about $7^{\prime \prime}$, while the solar chromosphere is only about $3^{\prime \prime}$ thick. Nevertheless, unprecedented spatial measurements can and have been achieved using the moments of X-ray distributions. The total flux (zeroth moment) from individual sources in various energy ranges has allowed us to perform imaging spectroscopy (e.g. Krucker \& Lin 2002; Emslie et al. 2003; Battaglia \& Benz 2007; Saint-Hilaire et al. 2008). The measurements of the X-ray source positions (first moments) pinpoint source locations with $1^{\prime \prime}$ or better accuracy and allow us to infer the chromospheric density structure (Aschwanden et al. 2002; Liu et al. 2006; Kontar et al. 2008b). The motions of HXR footpoint locations have been used to infer the reconnection rate in solar flares (Fletcher \& Hudson 2002; Krucker et al. 2003; Fivian et al. 2009). Using X-ray visibilities (Hurford et al. 2002; Schmahl et al. 2007) Kontar et al. (2008b) have measured not only the positions but the HXR footpoint sizes (second moment) at various energies and heights and found that HXR sources decrease with energy and consequently with height above the photosphere. Xu et al. (2008) have measured coronal sources to infer acceleration region sizes. HXR images can also be inverted (e.g. Brown et al. 2006) to find the spatial electron distributions and hence the locations of electron centroids (e.g. Prato et al. 2009).

Since the solar atmosphere above HXR sources is optically thin, X-rays are often directly related to the emitting electrons. However, the photons emitted downwards, toward the denser layers of the atmosphere interact with free or bound electrons and can also be scattered toward the observer (Tomblin 1972; Santangelo et al. 1973). Photons back-scattered and emerging back from the dense solar atmosphere to the observer create the albedo X-ray photons. Even for an isotropic X-ray source (the minimum albedo), the albedo flux can account for up to $40 \%$ of the detected flux in the range between 30 and $50 \mathrm{keV}$ (Bai \& Ramaty 1978; Zhang \& Huang 2004; Kontar et al. 2006; Kašparová et al. 2007). Therefore, all X-ray sources at the solar disk are viewed as a combination of both the primary and backscattered fluxes. Accounting for the albedo effect is important for all X-ray solar observations, which can only view disk sources as a combination of the primary photon flux and the backscattered photon flux. The backscattered component taints the primary source properties such as electron angular, energy, 
and spatial distributions. Albedo changes the shape of the spatially integrated X-ray spectrum, which is flattened at lower energies up to around $20-30 \mathrm{keV}$ and can even produce artificial spectral features in observed spectra (Kontar et al. 2008a), while at higher energies above around $70 \mathrm{keV}$, the the spectrum is steeper than the emitted (primary) spectrum. Kontar et al. (2006) have developed and implemented albedo correction for spectral X-ray RHESSI analysis using Green's functions approximations by Magdziarz \& Zdziarski (1995). Since the reflected X-rays come from a rather large area (albedo patch), the surface brightness of the albedo patch at the solar surface is rather low (Bai \& Ramaty 1978). This fact explains the difficulty in directly imaging the albedo patch (Schmahl \& Hurford 2002), but this highlights the importance of the inclusion of albedo for understanding the measurements of the source positions and sizes (first and second moments), the quantities which are integrated over the full area of the source.

In this Letter, using Monte Carlo simulations of X-ray photon transport we demonstrate how the observed positions and source sizes are affected by the albedo effect for various anisotropies, primary source sizes and primary source spectra and show that on-disk HXR sources should have energydependent minimum observed sizes.

\section{Spatial characteristics of the primary, backscattered and observed X-ray distributions}

The backscattered flux and albedo effect are studied using a Monte Carlo simulation starting with a hundred million photons per run. An unpolarized X-ray source was modeled in space with a 2-dimensional circular Gaussian $\sim \exp \left[-x^{2} /\left(2 d^{2}\right)-y^{2} /\left(2 d^{2}\right)\right]$ with width $d$, placed at the height $h=1 \mathrm{Mm}$ above the photosphere (the photosphere is defined here as a layer with hydrogen number density $1.16 \times 10^{17} \mathrm{~cm}^{-3}$ Vernazza et al. 1981). This is the typical hard X-ray source height found in footpoints (Aschwanden et al. 2002; Kontar et al. 2008b). The energy spectrum for photons has a power law $I(\epsilon) \sim \epsilon^{-\gamma}$ with a spectral index of $\gamma$, for energies between $3 \mathrm{keV}$ and $300 \mathrm{keV}$, typical for RHESSI. The code accounts for the curvature of the Sun and the photons are assumed to move freely until they reach the photospheric density at a height $z_{\odot}=\sqrt{R_{\odot}^{2}-x^{2}-y^{2}}-R_{\odot}$, where $R_{\odot}=6.96 \times 10^{10} \mathrm{~cm}$ is the solar radius. Below this level photons can be either scattered or photo-electrically absorbed. Similar to previous MC simulations (Bai \& Ramaty 1978; Magdziarz \& Zdziarski 1995), the Klein-Nishina cross-section for unpolarized $\mathrm{X}$-ray radiation was used

$\frac{\mathrm{d} \sigma_{\mathrm{c}}}{\mathrm{d} \Omega}\left(\epsilon_{0}, \theta_{\mathrm{s}}\right)=\frac{1}{2} r_{0}{ }^{2}\left(\left(\frac{\epsilon}{\epsilon_{0}}\right)^{3}+\frac{\epsilon}{\epsilon_{0}}-\left(\frac{\epsilon}{\epsilon_{0}}\right)^{2} \sin ^{2} \theta_{\mathrm{s}}\right)$,

where $\epsilon_{0}$ is the initial photon energy, $\epsilon$ is the new photon energy, $\theta_{\mathrm{s}}$ is the angle between the initial and new photon direction and $r_{0}=2.82 \times 10^{-13} \mathrm{~cm}$ is the classical electron radius. After a scattering, the new photon energy is just given by $\epsilon=\epsilon_{0} /\left(1+\frac{\epsilon_{0}}{m c^{2}}\left(1-\cos \theta_{\mathrm{s}}\right)\right)$. The absorption of X-ray photons, which is the dominant process below $\sim 10 \mathrm{keV}$ was modeled using modern solar photospheric abundances (Asplund et al. 2009) and cross-sections (Henke et al. 1982; Balucinska-Church \& McCammon 1992) for the most important elements $\mathrm{H}, \mathrm{He}, \mathrm{C}$, $\mathrm{N}, \mathrm{O}, \mathrm{Ne}, \mathrm{Na}, \mathrm{Mg}, \mathrm{Al}, \mathrm{Si}, \mathrm{S}, \mathrm{Cl}, \mathrm{Ar}, \mathrm{Ca}, \mathrm{Cr}, \mathrm{Fe}$ and Ni. For X-ray energies $>10 \mathrm{keV}$, photoelectric absorption was approximated as $\sigma_{\mathrm{a}}\left(\epsilon_{0}\right) \sim \epsilon_{0}^{-3}$ (Magdziarz \& Zdziarski 1995). To account for elements with more electrons than hydrogen, e.g. helium, carbon etc., Eq. (1) was multiplied by 1.18. Our simulations differ from previous simulations (e.g. Bai \& Ramaty 1978; Magdziarz \& Zdziarski 1995) because of newer abundances and the inclusion of the curvature of the Sun. The escaping photons are accumulated to create the brightness distribution $I(x, y)$ over a given energy and solid angle. The total primary or reflected flux is then just an integral over the corresponding area $\int I(x, y) \mathrm{d} x \mathrm{~d} y$. Figure 1a shows the primary and escaping photon brightness distributions for a source located at the disk centre. Similar to the previous results (Bai \& Ramaty 1978) we see that for a compact primary source of size $d=1.5 h$, the back-scattered (albedo) photons are reflected from an area much larger than the primary source. The reflected photons change the spatial distribution of the observed photons and produce a halo around the primary source. Importantly, even a primary point source will be seen as a source of finite size (Fig. 2). The brightness distribution of a large primary source of $d=4.5 h$ is less influenced by the reflected photons but nevertheless the source will look larger than it actually is.

Using solar disk centered coordinates, the centroid position of the source $(\bar{x}, \bar{y})$ can be found by calculating the first normalized moment of the distribution (mean)

$\bar{x}=\frac{\int_{-\infty}^{\infty} x I(x, y) \mathrm{d} x \mathrm{~d} y}{\int_{-\infty}^{\infty} I(x, y) \mathrm{d} x \mathrm{~d} y} \quad, \quad \bar{y}=\frac{\int_{-\infty}^{\infty} y I(x, y) \mathrm{d} x \mathrm{~d} y}{\int_{-\infty}^{\infty} I(x, y) \mathrm{d} x \mathrm{~d} y}$

and the normalized variance of the distribution (second moment),

$\sigma_{x}^{2}=\frac{\int_{-\infty}^{\infty}(x-\bar{x})^{2} I(x, y) \mathrm{d} x \mathrm{~d} y}{\int_{-\infty}^{\infty} I(x, y) \mathrm{d} x \mathrm{~d} y}, \sigma_{y}^{2}=\frac{\int_{-\infty}^{\infty}(y-\bar{y})^{2} I(x, y) \mathrm{d} x \mathrm{~d} y}{\int_{-\infty}^{\infty} I(x, y) \mathrm{d} x \mathrm{~d} y}$

Hereafter, following RHESSI measurements (Kontar et al. 2008b; Dennis \& Pernak 2009; Prato et al. 2009) we will refer to the source sizes in terms of FWHM (Full Width Half Maximum), $F W H M_{x, y}=2 \sqrt{2 \ln 2} \sigma_{x, y}$.

The scattered X-ray flux depends on the cosine of the heliocentric angle of the source $(\mu \equiv \cos (\theta))$ or equivalently on the position of the source at the solar disk, $\mu=\sqrt{1-\left(x^{2}+y^{2}\right) / R_{\odot}^{2}}$. A circular X-ray source located above the centre of the disk will produce a circular albedo patch (Fig. 1a). Naturally, the location of the HXR source and albedo patch will coincide at the disk centre, so albedo will not change the source position. However, the albedo will make the source larger than it is actually is (Fig. 1a). The albedo contribution becomes asymmetric if the source is located away from the disk centre at a given heliocentric angle $\theta$ (Figs. 1b-d). Due to the spherical symmetry of the Sun, there are two distinct directions: radial - along the line connecting the centre of the Sun and the X-ray source $r$, and perpendicular to the radial $r_{\perp}$. There is no change in centroid position in the $r_{\perp}$-direction for a spherically symmetric primary source. In the $r$-direction, the albedo causes a centroid shift towards the disk centre that rises from $\sim 0$ at $\mu=1.0$ and peaks shortly before falling to $\sim 0$ again at $\mu=0.0$. Figure 1 also shows how the source size varies in the $r_{\perp}$ direction, with the $F W H M$ of the source generally decreasing at lower $\mu$. In the radial direction, the FWHM of the total and primary sources decreases close to linear due to a simple projection effect. The detailed 3D structure of the source is required before any physically meaningful predictions can be made concerning the change in source size in the radial direction, and this is beyond the scope of the paper. Therefore, we consider the source sizes in the $r_{\perp}$ direction and the source position in the radial direction rather than along the 
E. P. Kontar and N. L. S. Jeffrey: Positions and sizes of X-ray solar flare sources
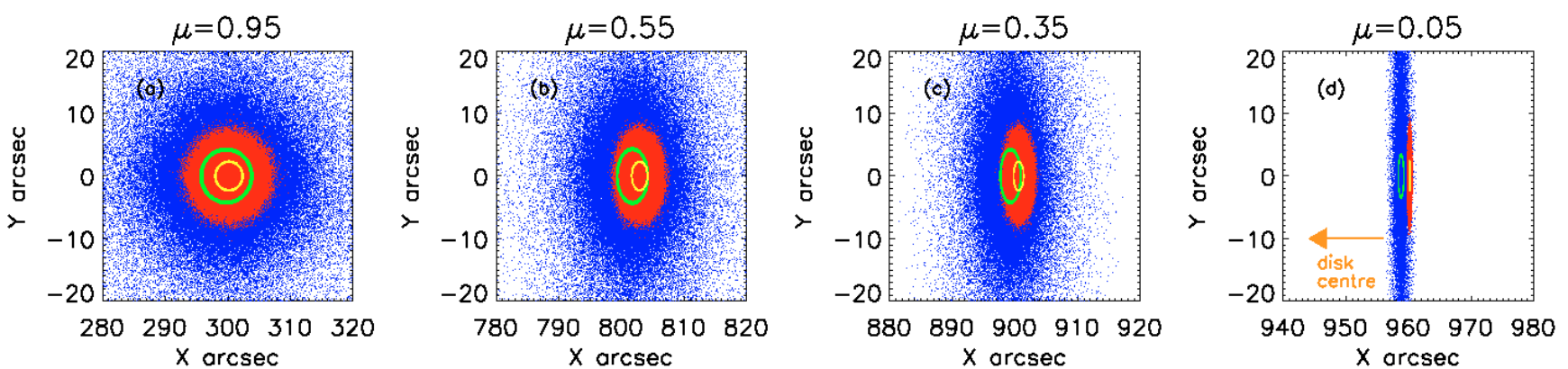

Fig. 1. The X-ray scatter distributions of the primary photons (red dots) and the Compton back-scattered photons (blue dots) for a primary source at $h=1.0 \mathrm{Mm}$ with $d=1.5 \mathrm{Mm}\left(F W H M \sim 4.9^{\prime \prime}\right)$ between 20 and $50 \mathrm{keV}$ for four viewing angles given by $\mu$. The yellow and green ellipses show the $F W H M$ sizes for the primary and combined sources respectively.


Fig. 2. Spectral index dependency (panels a)-d)): the source position shift is in the radial direction due to albedo (source $\mu=0.55$ ) and source size $F W H M$ is in the perpendicular to radial direction for various spectral indices $\gamma$ for an isotropic source with $F W H M \sim 4.9^{\prime \prime}:$ green $-\gamma=4$; red $-\gamma=3$; purple $-\gamma=2$. Primary source size dependency (panels $\mathbf{e})-\mathbf{h})$ ): isotropic primary source with $\gamma=3$ : orange - point source, red $F W H M \sim 4.9^{\prime \prime}$, blue $-F W H M \sim 14.6^{\prime \prime}$. Anisotropy dependency (ratio of downward to upward directed fluxes) (panels i)-1)): simulations are for the primary source with $F W H M \sim 4.9^{\prime \prime}$ and spectral index $\gamma=3$ : red - anisotropy $=1$ (isotropic), blue - anisotropy $=2$, green - anisotropy $=5$. Graphs as a function of energy are for $\mu=0.55$ and the graphs as a function of $\mu$ are for energies between 20 and $50 \mathrm{keV}$.

East-West and South-North directions. Similar to the spatially integrated albedo (Kontar et al. 2006), the shift in centroid position and the growth of the source are also energy and $\mu$ dependent. In the following, we consider the position and source size changes for various a) spectra of the primary source, b) primary source size, and c) X-ray directivity (the ratio of downward to upward emitted photons) separately. The results are summarized in Fig. 2.

Spectral index (Figs. 2a-d) - similar to the spectral results, the albedo contribution from a smaller spectral index produces the largest shift in position and a larger total source size (Figs. $2 \mathrm{a}-\mathrm{d}$ ). An isotropic source of $F W H M \sim 4.9^{\prime \prime}$ for the lowest modeled spectral index of $\gamma=2$ produces the greatest shift of $\sim 0.5^{\prime \prime}$ at $\mu=0.5-0.6$ and $\sim 30 \mathrm{keV}$. This spectral index also produces the largest source size and has a $F W H M \sim 9.5^{\prime \prime}$ at $\mu=1.0$, compared with the other spectral indices of $\gamma=3,4$ modeled.
Primary source size (Figs. 2e-h) - for a fixed spectral index of $\gamma=3$, all primary source sizes produce the same shift in centroid position. The maximum shift in position occurs at $\mu=$ 0.5-0.6 and $\sim 30 \mathrm{keV}$ for all sources (Figs. 2e, g). Although the $F W H M$ of the total source grows with increasing primary size, it is observed that the relative size of the total to the primary source is smaller for a larger primary source. This indicates that a larger primary source should have a smaller relative size increase due to albedo. Even an initial point source produces a total source with a FWHM peaking around 7" (Figs. 2f, h).

Anisotropy (Figs. 2i-l) - the shift in centroid position is larger for a higher initial downward anisotropy (the ratio of downward flux to upward flux) for all $\mu$ and energies (Figs. 2i, k). All shifts follow the general trend and tend towards zero at the centre $(\mu=1.0)$ and the limb $(\mu=0.0)$. Using $\gamma=3$ and a primary source of $F W H M \sim 4.9^{\prime \prime}$, a directivity of 5 produces a 
peak difference of $\sim 0.9^{\prime \prime}$ and even an isotropic source produces a peak difference of $\sim 0.4^{\prime \prime}$. The shift in source position peaks near $\mu=0.4-0.6$ and $\sim 30 \mathrm{keV}$ for a downward anisotropy of 2 and an isotropic source, but the shift peaks at a lower $\mu=0.4-0.5$ for a downward directivity of 5 . The stronger downward beaming of the primary source also leads to larger apparent source sizes for all $\mu$ and energies (Figs. $2 \mathrm{j}, 1$ ). It should be observed that the total $F W H M$ produced for a directivity of 5 peaks at $\mu \sim 0.15$ (Fig. 2p) giving an apparent $F W H M \sim 13^{\prime \prime}$. Since the fraction of reflected photons reduces with $\mu$ the $F W H M$ in perpendicular direction can be expected to slowly decrease from disk centre to limb, but the $F W H M$ actually increases, peaks at $\mu \sim 0.15$ and only then starts to decrease. This effect is due to the angular dependence of the Compton cross-section. The cross-section is anisotropic and peaks at $90^{\circ}$, which allows a larger number of photons to scatter into an observer direction for flares close to the limb. It is this anisotropy in the scattering of the photons that causes the $F W H M$ to peak at an angle smaller than $\mu=1.0$. The observation of this effect is particularly clear in the case of high downward directivity (Fig. 21).

\section{Discussion and conclusions}

The results of the simulations show that albedo can substantially affect the precise position and source size measurements of X-ray sources. Therefore, the effect of albedo should always be (probably with the exception of limb/occulted flares) considered when the sizes or positions of X-ray sources are analyzed. The albedo displacement of the source position is radially directed towards the disk centre and depends on the anisotropy of $\mathrm{X}$-ray radiation, the $\mathrm{X}$-ray source size and the spectral index of the primary source. Similar to total reflected flux, the displacement of HXR source position is energy dependent. The largest displacement can be observed in the range between $30-50 \mathrm{keV}$ at $\mu \sim 0.5$ (heliocentric angle $\sim 60^{\circ}$ ). The shift in centroid position in this energy range is $0.1-0.5^{\prime \prime}$ for an isotropic (minimum albedo) source 1.4" above the photosphere and this can be up to $\sim 0.9^{\prime \prime}$ for a downward beaming with factor of 5 . Because of the albedo, X-ray source sizes will be energy dependent, larger in the perpendicular to radial direction, and elliptical even for a spherically symmetric primary source. In the perpendicular to radial direction, the largest growth in source size occurs for sources close to the solar disk centre, in the energy range between $30-50 \mathrm{keV}$, where albedo is the strongest. Thus, an isotropic primary source with $F W H M \sim 4.9^{\prime \prime}$ at $1.4^{\prime \prime}$ above the photosphere will have an apparent $F W H M$ size of $\sim 9^{\prime \prime}$ in the energy range $20-50 \mathrm{keV}$ for sources in the wide range of heliocentric angles from $0^{\circ}$ to $\sim 80^{\circ}$.

The simulations demonstrate that X-ray sources will have a minimum size. An isotropic point source at $1.0 \mathrm{Mm}$ above the photosphere will be measured by RHESSI as a source with a $F W H M$ size of $\sim 7^{\prime \prime}$ across. This result can explain larger X-ray footpoint sizes than EUV or optical ones (e.g. Kašparová et al. 2005). Dennis \& Pernak (2009) reported that the average semiminor axis of 18 double source flares is about $4^{\prime \prime}$, while a few of the X-ray source sizes were found to be consistent with line sources along the flare ribbons. While the quantitative comparison with the RHESSI observations requires additional work, we note that zero sizes are either the artifacts of the algorithms used or are caused by the very low source heights.

The energy dependent character of albedo predicts that the source size as measured by RHESSI should grow with energy from $10 \mathrm{keV}$ up to $\sim 30 \mathrm{keV}$. Considering a large primary source of 14.6" across, e.g. a flaring loop, we find that the source will grow up to $\sim 18^{\prime \prime}$ at $\sim 30 \mathrm{keV}$. Noteworthy, Xu et al. (2008) have found that coronal source sizes are growing with energy along both the field lines and across. While the field line increase along the lines could be an indicator of electron transport or of the acceleration region size, the cross-field increase remains unexplained, but is consistent with the growth of the source size due to the albedo. We note that the spatial changes of X-ray sources due to albedo have a great diagnostic potential for purely known anisotropy of energetic electrons.

Acknowledgements. The authors are indebt to G. Hurford for insightful comments. E.P.K. work is supported by a STFC rolling grant, STFC Advanced Fellowship and the Leverhulme Trust grant. N.L.S.J. work was supported by The Nuffield Foundation and Cormack Bequest, Royal Society Edinburgh. The work has benefited from the international team grant from ISSI, Bern, Switzerland.

\section{References}

Aschwanden, M. J., Brown, J. C., \& Kontar, E. P. 2002, Sol. Phys., 210, 383 Asplund, M., Grevesse, N., Sauval, A. J., \& Scott, P. 2009, ARA\&A, 47, 481 Bai, T., \& Ramaty, R. 1978, ApJ, 219, 705

Balucinska-Church, M., \& McCammon, D. 1992, ApJ, 400, 699

Battaglia, M., \& Benz, A. O. 2007, A\&A, 466, 713

Brown, J. C., Emslie, A. G., Holman, G. D., et al. 2006, ApJ, 643, 523

Dennis, B. R., \& Pernak, R. L. 2009, ApJ, 698, 2131

Emslie, A. G., Kontar, E. P., Krucker, S., \& Lin, R. P. 2003, ApJ, 595, L107

Fivian, M. D., Krucker, S., \& Lin, R. P. 2009, ApJ, 698, L6

Fletcher, L., \& Hudson, H. S. 2002, Sol. Phys., 210, 307

Henke, B. L., Lee, P., Tanaka, T. J., Shimabukuro, R. L., \& Fujikawa, B. K. 1982, Atomic Data and Nuclear Data Tables, 27, 1

Hurford, G. J., Schmahl, E. J., Schwartz, R. A., et al. 2002, Sol. Phys., 210, 61 Kašparová, J., Karlický, M., Kontar, E. P., Schwartz, R. A., \& Dennis, B. R. 2005, Sol. Phys., 232, 63

Kašparová, J., Kontar, E. P., \& Brown, J. C. 2007, A\&A, 466, 705

Kontar, E. P., Dickson, E., \& Kašparová, J. 2008a, Sol. Phys., 252, 139

Kontar, E. P., Hannah, I. G., \& MacKinnon, A. L. 2008b, A\&A, 489, L57

Kontar, E. P., MacKinnon, A. L., Schwartz, R. A., \& Brown, J. C. 2006, A\&A, 446, 1157

Kosugi, T., Masuda, S., Makishima, K., et al. 1991, Sol. Phys., 136, 17

Krucker, S., \& Lin, R. P. 2002, Sol. Phys., 210, 229

Krucker, S., Hurford, G. J., \& Lin, R. P. 2003, ApJ, 595, L103

Lin, R. P., Dennis, B. R., Hurford, G. J., et al. 2002, Sol. Phys., 210, 3

Liu, W., Liu, S., Jiang, Y. W., \& Petrosian, V. 2006, ApJ, 649, 1124

Magdziarz, P., \& Zdziarski, A. A. 1995, MNRAS, 273, 837

Prato, M., Emslie, A. G., Kontar, E. P., Massone, A. M., \& Piana, M. 2009, ApJ, 706, 917

Saint-Hilaire, P., Krucker, S., \& Lin, R. P. 2008, Sol. Phys., 250, 53

Santangelo, N., Horstman, H., \& Horstman-Moretti, E. 1973, Sol. Phys., 29, 143

Schmahl, E. J., \& Hurford, G. J. 2002, Sol. Phys., 210, 273

Schmahl, E. J., Pernak, R. L., Hurford, G. J., Lee, J., \& Bong, S. 2007, Sol. Phys., 240,241

Tomblin, F. F. 1972, ApJ, 171, 377

Vernazza, J. E., Avrett, E. H., \& Loeser, R. 1981, ApJS, 45, 635

Xu, Y., Emslie, A. G., \& Hurford, G. J. 2008, ApJ, 673, 576

Zhang, J., \& Huang, G. L. 2004, Sol. Phys., 219, 135 